\title{
A shape memory porous sponge with tunability in both surface wettability and pore size for smart molecule release
}

\author{
Pengchang Liu ${ }^{1 \dagger}$, Hua Lai ${ }^{1 \dagger}$, Qixing Xia ${ }^{3}$, Dongjie Zhang ${ }^{1}$, Zhongjun Cheng ${ }^{1 *}$, Yuyan Liu ${ }^{1 *}$ and \\ Lei Jiang ${ }^{2}$
}

\begin{abstract}
Recently, smart superwetting porous materials have aroused much attention, and it is well known that tunable surface wettability and pore structure/size are extremely important for their functions. However, only one factor can be regulated on existing materials, which significantly restricts their controllability, functions, and applications. A new material was prepared by electrodepositing a layer of polypyrrole on a shape memory sponge, on which switchable superhydrophobicity/superhydrophilicity and adjustable pore size within the range of about $28 \mathrm{~nm}$ to $895 \mu \mathrm{m}$ can be observed. Through synergistically tuning the wettability and pore size, both ON/OFF water permeation and accurate flux can be obtained. Meanwhile, we also applied the sponge for molecule release, and diverse release manners with precise/smart controllability can be accomplished. This paper reports for the first time a smart material with controllability in both surface wetting and pore size, which provides a new strategy for the preparation of novel smart superwetting porous materials.
\end{abstract}

Keywords: shape memory porous sponge, surface wettability, pore size, smart molecule release

\section{INTRODUCTION}

Nowadays, because of the tunable wettability and porous structure, smart superwetting porous materials have been extensively studied and applied in many areas [1,2], such as oil/water emulsion separation [3-5], underwater gas manipulation [6-8], drug release [9-11], and electrocatalysis $[12,13]$. The most common examples of such smart materials are those with switchable (super-)hydrophobicity/(super-)hydrophilicity [14-16], which are fabricated by modifying responsive molecules on static porous substrates $[17,18]$, for instance, $\operatorname{poly}(N$-isopropylacrylamide) with temperature responsibility coated on nylon membrane [19], light-responsive spiropyranmodified mesoporous silica nanoparticles [20], and some kinds of stimuli-responsive systems, including $\mathrm{pH}$ [2123], electric field [24,25], gas [26], and ions [27]. Besides switchable surface wettability, the pore structure/size is also crucial for material's function and application, and elastic polydimethylsiloxane (PDMS) porous films were often used as the substrate to adjust pore size under repeatedly stretching/releasing [28]. However, as to all these reports, surface wettability or pore size can be changed singly; materials with simultaneously adjustable surface wettability and pore size have not been reported yet.

As to the superwetting porous materials, controllable water permeation is important because it can directly influence the material's application in many areas [29-36] such as molecule release [9-11,34], precise chemical synthesis [35,36], and oil-water separation [31-33]. However, existing superwetting porous materials obtained from current designing strategies still hardly meet the increasing demand in practical applications. For instance, as to these materials with switchable wettability [30], only ON/OFF water permeation can be observed, since all these porous materials have a constant pore structure/size, and precise permeation flux is difficult to

\footnotetext{
${ }^{1}$ State Key Laboratory of Urban Water Resource \& Environment, School of Chemistry and Chemical Engineering, Harbin Institute of Technology, Harbin 150001, China

${ }^{2}$ CAS Key Laboratory of Bio-inspired Materials and Interfacial Science, Technical Institute of Physics and Chemistry, Chinese Academy of Sciences, Beijing 100190, China

${ }^{3}$ Institute of Culture and Heritage, Northwest Polytechnical University, Xi'an 710000, China

$\dagger$ These authors contributed equally to this work.

* Corresponding authors (emails: chengzhongjun@iccas.ac.cn (Cheng Z); liuyy@hit.edu.cn (Liu Y))
} 
be achieved. This problem also limits their applications in accurate molecule release and precise chemical synthesis that have a high requirement in accurate release velocity. For the elastic PDMS porous film, due to the intrinsic hydrophobicity, it is not convenient to adjust the water permeation. As reported by Hou and co-works [28], a steady-state external force is necessary to realize the permeation control, which is unsuitable for those applications where liquid permeation can occurr spontaneously, such as drug release and gravity driving oil/water separation. Meanwhile, the pore size is changed as the external pressure is removed. Recently, to solve this problem, we fabricated a superhydrophilic shape memory sponge (SMS) [37], which can memorize diverse pore sizes and thus realize the permeation flux control. However, such an SMS cannot display the ON/OFF permeation control as the pore size is constant. From the above, it can be found that current porous materials based on singly adjusting surface wettability or pore size can hardly meet the increased requirement of smart/precise water permeation control. A new superwetting porous material that has a tunable pore size, and meanwhile can demonstrate the ON/OFF permeation control under a random pore size would be more perfect. Such a porous material would be promising to overcome all the present shortcomings, and provide better controllability and new functions; however, until now, this is still a challenge.

Herein, we advance a new design method by integrating a superhydrophilic SMS with the polypyrrole (PPy) film doped with perfluorooctanesulfonate (PFOS) ions, and report such a porous material (Fig. 1). PFOS ion-doped PPy offers switchable hydrophobicity/hydrophilicity by alternating the applied redox potentials (Fig. 1a), and SMS provides the tunable pore size because of the shape memory effect (SME, Fig. 1b). Through combining different wetting performances and pore sizes, diverse states of the sponge can be obtained and reversibly controlled between each other (Fig. 1c-f). Due to the excellent

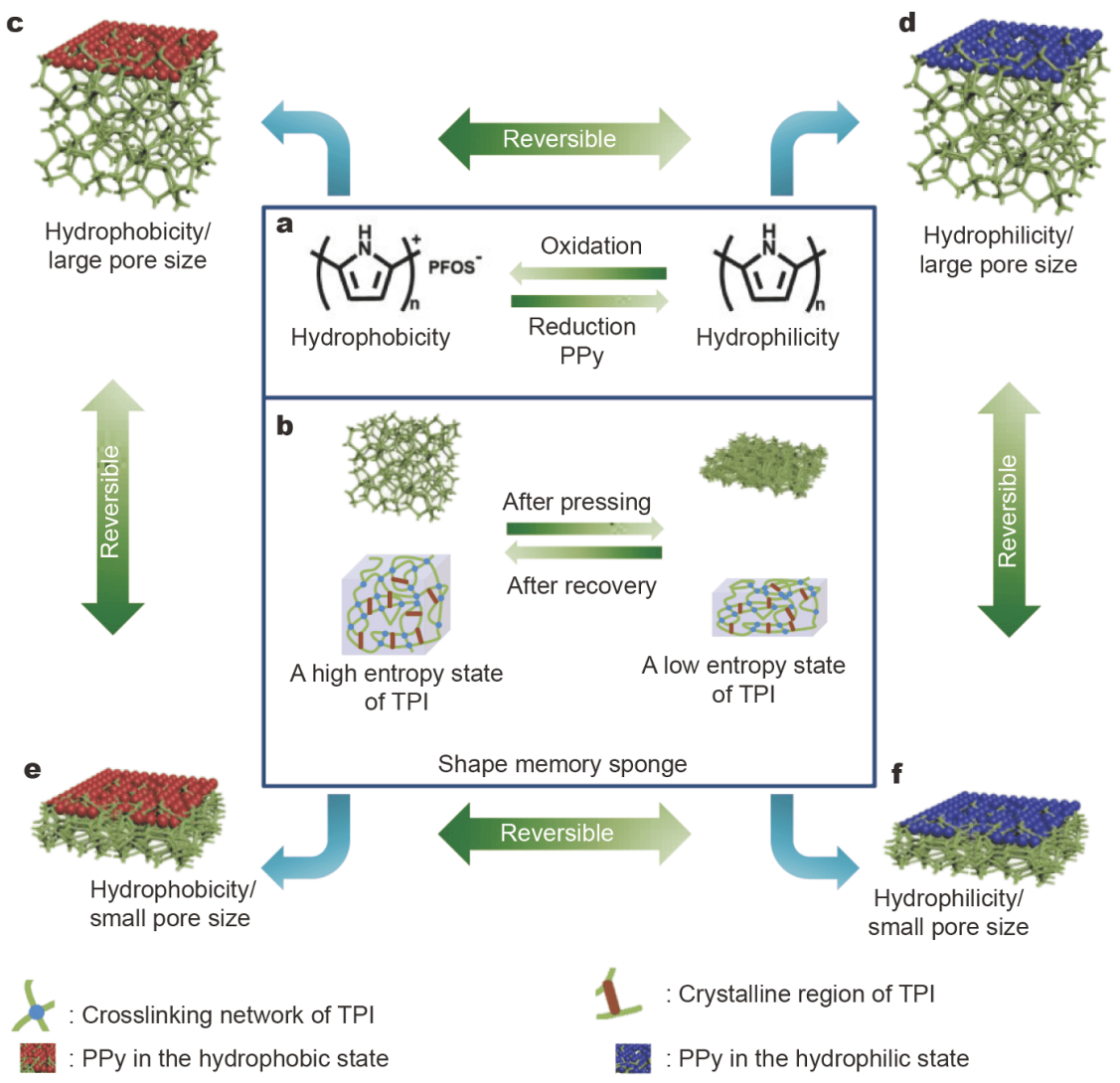

Figure 1 Schematic illustration of the design principle and smart control of the sponge. (a) PFOS ion-doped PPy that can transit between hydrophobicity/hydrophilicity was used to coat the top surface of the sponge to provide switchable wetting performance. (b) Shape memory polymer TPI-coated sponge was used as the substrate to offer the tunable pore size. By synergistically adjusting both the surface wettability and pore size, the sponge can be smartly controlled between multiple states: (c) large pores with hydrophobic surface; (d) large pores with hydrophilic surface; (e) small pores with hydrophobic surface; and (f) small pores with hydrophilic surface, respectively. 
controllability in both surface wetting performance and pore size, ON/OFF water permeation with simultaneous precise permeation flux can be achieved. Meanwhile, derived from the smart controllability of the SMS, the SMS was further applied in molecule release and diverse release manners with smart/precise controllability were also achieved. This paper first develops a superwetting porous material with tunability in both surface wettability and pore size, which offers a strategy for designing new superwetting porous materials, and meanwhile can be potentially applied in many research areas, such as molecule release, accurate chemical synthesis, and microfluidic devices.

\section{EXPERIMENTAL SECTION}

\section{Fabrication of the superhydrophilic SMS}

The preparation of SMS is similar to previous report [37]. Briefly, trans-1,4-polyisoprene (TPI) and dicumyl peroxide (DCP, weight ratio was 100:2) were firstly dissolved in chloroform $\left(20 \mathrm{mg} \mathrm{mL}^{-1}\right)$ at room temperature. Then, the clean polyurethane (PU) sponge was immersed in the above solution for about $0.5 \mathrm{~h}$ and taken out for drying overnight. In a nitrogen atmosphere, the sponge was further heated at $160^{\circ} \mathrm{C}$ for approximately $15 \mathrm{~min}$ to complete the cross-linking chain reaction. Finally, we can obtain the sponge with SME since the PU sponge framework has been covered by the TPI coating. The superhydrophilic SMS was synthesized via polydopamine/ polyethylenimine (PDA/PEI) deposition. Dopamine hydrochloride (DA, 98\%) and PEI $\left(M_{\mathrm{w}}: 600\right)$ (mass ratio was 1:1) were dissolved in Tris- $\mathrm{HCl}(\mathrm{pH}$ 8.5, $\left.20 \mathrm{mmol} \mathrm{L}^{-1}\right)$ to obtain a DA/PEI solution $\left(2 \mathrm{mg} \mathrm{L}^{-1}\right)$. After magnetic stirring the solution containing the SMS for about $12 \mathrm{~h}$ and then drying the SMS in vacuum at $60^{\circ} \mathrm{C}$ overnight, the superhydrophilic SMS was obtained.

\section{Fabrication of the PPy coating on the SMS}

The gold nanoparticles on top surface of the SMS were firstly synthesized by ion sputtering a gold target in vacuum. Then, tetraethylammonium PFOS-doped PPy coating was galvanostatically polymerized on the top surface of the SMS at a fixed current density of $0.25 \mathrm{~mA} \mathrm{~cm}^{-2}$ by using a CHI 760 electrochemical workstation. The three-electrode system was used for electrochemical polymerization. The SMS, $\mathrm{Ag} / \mathrm{AgCl}$ electrode $\left(3.5 \mathrm{~mol} \mathrm{~L}^{-1} \mathrm{KCl}\right)$, and a platinum foil were used as the working electrode, reference electrode and counter electrode, respectively. The electrolyte solution contained $0.1 \mathrm{~mol} \mathrm{~L}^{-1}$ pyrrole, $5 \times 10^{-4} \mathrm{~mol} \mathrm{~L}^{-1} \mathrm{FeCl}_{3}$, and
$0.02 \mathrm{~mol} \mathrm{~L}^{-1}$ PFOS in acetonitrile. After the electrochemical polymerization for about $3600 \mathrm{~s}$, the SMS was washed in acetonitrile to remove the impurities and dried at $70^{\circ} \mathrm{C}$ overnight.

\section{Wettability switching experiments}

Electricity-controlled wettability switching experiments was conducted using the same device for the preparation of the PPy coating. The PPy coating can be converted from the oxidation state into the reductive state at a negative electric potential $(-0.6 \mathrm{~V})$ for about $3600 \mathrm{~s}$. After being treated at a positive electric potential $(1.0 \mathrm{~V})$ for about $3600 \mathrm{~s}$, the oxidation state can be regained. After each transition, the sponge needs to be washed with acetonitrile to remove the impurities and dried at $70^{\circ} \mathrm{C}$ overnight.

\section{Control of the shape recovery degree $\left(R_{s}\right)$ of the sponge and the calculation of the $R_{\mathrm{s}}$}

The shape recovery degree $\left(R_{\mathrm{s}}\right)$ was defined to represent a random state of the SMS from the pressed state to the recovery state. The initial thickness of the SMS was defined as $h_{0}$. In order to prepare the SMS with different $R_{s}$, the obtained SMS was heated and pressed at $70^{\circ} \mathrm{C}$ and cooled under external force (about $4.5 \times 10^{3} \mathrm{~Pa}$ ) at $25^{\circ} \mathrm{C}$. The pressed state can be memorized and defined as the pressed state with $R_{\mathrm{s}}=0 \%$. A mold with two parallel plates was used to control the thickness of SMS in the recovery process (Fig. S1), based on which, the thickness of the SMS can be adjusted accurately. By tuning the space between the two plates, sponge with diverse $R_{\mathrm{s}}$ can be obtained. The calculation of the $R_{\mathrm{s}}$ is according to the following equation:

$R_{\mathrm{s}}=\frac{h_{x}-h_{1}}{h_{0}-h_{1}} \times 100 \%$,

where $h_{1}$ is the thickness of SMS at the pressed state, and $h_{x}$ is the thickness of SMS at a random state. Specifically, in this work, $h_{0}=3 \mathrm{~cm}, h_{1}=0.5 \mathrm{~cm}$, and the space between two parallel plates was set as $3,2.5,2,1.5$ and $1 \mathrm{~cm}$, respectively. The corresponding $R_{\mathrm{s}}$ values were obtained to be $100 \%, 80 \%, 60 \%, 40 \%$ and $20 \%$, respectively.

\section{Characterization}

The morphologies were observed using a scanning electron microscope (SEM; HITACHI SU 8010, Japan), and the Micro-XCT (Xradia Inc., USA). The water contact angels (WCAs) were measured by a contact angle measure meter system (JC 2000D5, Shanghai Zhongchen Digital Technology Apparatus Co., Ltd., China). The preparation of the gold nanoparticle film was conducted 
by the ion sputtering (LEICA EM SCD050). The ultraviolet-visible (UV-Vis) absorption spectra were measured on a PerkinElmer spectrophotometer (Lambda 750S, USA). The photographs in Fig. 2a were recorded by a camera (iPhone 7).

\section{RESULTS AND DISCUSSION}

Shape memory polymers (SMPs) have aroused increased attention since they can memorize and demonstrate diverse macro/micro-scale shapes [38-40], and thus realized numerous novel applications in biomedicine [41-
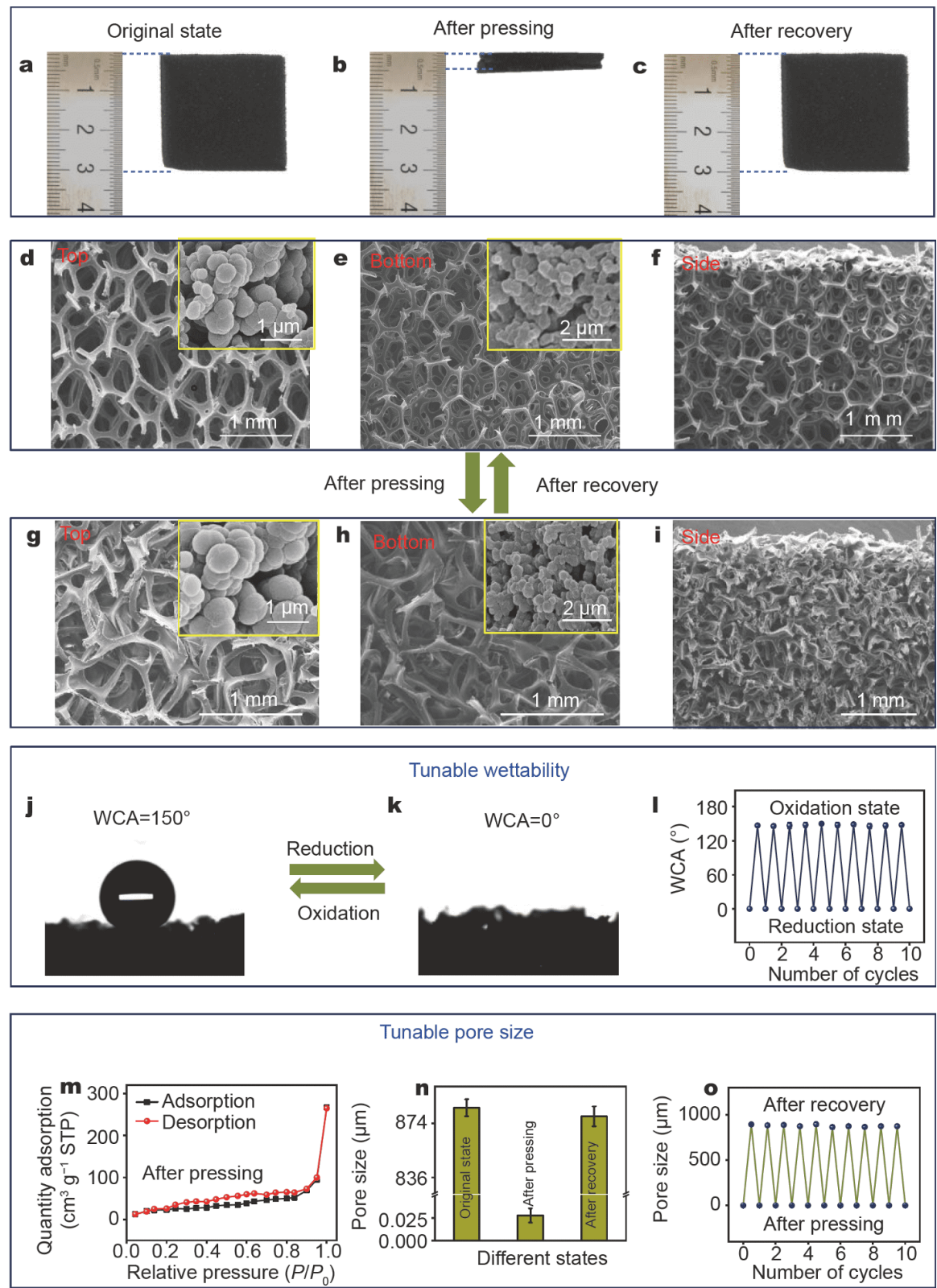

Figure 2 Photos of the as-prepared sponge at initial state (a), the pressed state (b), and the recovered state (c), respectively. (d-f) SEM images of the obtained sponge viewed from top, bottom and side, respectively. Insets are amplified images of the surface corresponding to (d) and (e), respectively. (g-i) SEM images of the sponge at the pressed state viewed from top, bottom, and side, respectively. Insets are magnified surface images corresponding to $(\mathrm{g})$ and $(\mathrm{h})$, respectively. $(\mathrm{j}, \mathrm{k})$ Shapes of a water droplet on the top surface when the PPy was alternately changed between the oxidation/ reduction states, indicating superhydrophobicity/superhydrophilicity switching can be achieved. (l) The top surface wetting performance can be repeatedly regulated. (m) Nitrogen adsorption-desorption isotherms of the SMS at the pressed state. (n) Statistic of the average pore size for the SMS in different states. (o) The pore size can be repeatedly tuned by alternately pressing/recovering the SMS's shape. The results confirm that the asprepared sponge has a good SME, and both surface wettability and pore size can be smartly controlled. 
43], optic chips [44,45], adhesive devices [46-48], and superwetting droplet manipulation platform, etc. [49-51]. Recently, the integration of the shape memory property with porous sponge has been tried to control the pore structure. Research results indicate that the obtained SMS can memorize and display diverse shapes and pore structures without any external assistance, which is apparently superior to those elastic PDMS porous films. For instance, through controlling the shape of oil-infused graphene SMS between the compressed/recovered states, Zhao and co-workers [24] developed controllable droplet sliding; by using a carbon nanotube SMS surface, $\mathrm{Wu}$ and co-workers [52] achieved the separation of oil and water since the SMS can spontaneously recover from the compressed state to the original state when it contacts liquid; based on an SMS whose shape can be spontaneously shrunk after heating, Bai and co-workers [53] reported fast absorption/self-squeezing of oils by using the smart SMS. Although so many SMSs have been prepared, none of them is suitable for controlling water penetration because their pore structures have been infused by oil, or the distorted pores cannot be preserved once they meet water. To solve this problem, we prepared a new superhydrophilic SMS, which was obtained by successively covering SMP TPI and hydrophilic PDA/PEI on a PU sponge framework (Figs S2-S6). After obtaining the superhydrophilic SMS, a layer of PFOS ion-doped PPy was further deposited on the top surface of the SMS (Figs S7 and S8). Fig. 2a shows the optical photograph of the as-prepared sponge, confirming that it has a thickness of approximately $3 \mathrm{~cm}$. The SEM images measured from top (Fig. 2d), bottom (Fig. 2e), and side (Fig. 2f) confirm that the mean pore size of the three-dimensional (3D) porous network structure is about $895 \mu \mathrm{m}$. Magnified pictures of top and bottom surfaces display that many nanoparticles with the size of approximately $800 \mathrm{~nm}$ (inset in Fig. 2d), and $500 \mathrm{~nm}$ (inset in Fig. 2e), respectively, are dispersed on corresponding surfaces, which are ascribed to PPy, and PDA/PEI, respectively. After being pressed at $70^{\circ} \mathrm{C}$ and cooled under external force at $25^{\circ} \mathrm{C}$, the pressed state of SMS was memorized and the thickness was changed to approximately $0.5 \mathrm{~cm}$ (Fig. 2b). SEM images viewed from all directions show that the micropores become unclear in the pressed state (Fig. 2g-i). Amplified images display that the PPy and PDA/PEI nanoparticles are still present on the surface, meaning that the pressing has no remarkable effect on these nanoparticles. Further reheating the SMS at $70^{\circ} \mathrm{C}$ for about $60 \mathrm{~s}$, it can be seen that the SMS can spontaneously return to the original state in both macroscopic and microscopic levels (Fig. 2c), demonstrating that the synthesized SMS has a good shape memory performance.

It has been widely reported that the surface wettability of the PFOS ion-doped PPy coating can be adjusted reversibly. On our SMS, similar results can also be observed. One can find that the top surface's WCA on the obtained SMS is about $150^{\circ}$, meaning that the SMS surface is superhydrophobic (Fig. 2j). As a reduction potential was applied for about $60 \mathrm{~min}$, the top surface becomes superhydrophilic with a WCA of about $0^{\circ}$ (Fig. 2k and Fig. S9a). Further applying an oxidation potential for about $60 \mathrm{~min}$, surface superhydrophobicity can be regained (Fig. S9b), and such superhydrophobic/ superhydrophilic transitions can be achieved as the oxidation and reduction potentials are alternately applied (Fig. 21). In addition to the switchable wettability, it is found that based on the excellent SME, the pore size of the SMS can also be reversibly changed. Fig. $2 \mathrm{~m}$ and Fig. S10 display the nitrogen adsorption-desorption isotherms of the sponge at different states. The presence of the hysteresis loop proves that the SMS can always keep the pore structure as the shape is changed [54]. During the same examination, the pore sizes of the SMS in original state, pressed state, and recovered state were also investigated, which are about $895 \mu \mathrm{m}, 28 \mathrm{~nm}$, and $895 \mu \mathrm{m}$, respectively (Fig. $2 \mathrm{n}$ and Fig. S11), indicating that SME allows the sponge to memorize different pore sizes, and the pore size can be regulated in a wide range from hundreds of micro-meters to dozens of nanometers. Together with the good mechanical strength of the sponge (Fig. S12), it can be found that alternative variation of the pore size can be repeated for several times (Fig. 20). Moreover, it should be emphasized that the application of oxidation/reduction potentials have no effect on the material's shape memory property, and meanwhile, the shape variation also has no influence on the smart wettability of the PPy. Even after 20 times of oxidation/reduction and pressing/recovering processes, responsive PPy and hydrophilic PDA/PEI nanoparticles are still present on the sponge surface (Fig. S13), further confirming the good stability of the smart SMS.

The key difference from common superwetting sponge is that our sponge has a good SME that can bring a tunable pore structure/size. In fact, in addition to the two states mentioned above (pressed and original states), the SMS can also memorize several other states. Herein, sponge can display diverse states with different $R_{\mathrm{s}}$ (the calculation of $R_{\mathrm{s}}$ is shown in EXPERIMENTAL SECTION and Fig. S1). As shown in Fig. S14, by directly controlling the $R_{\mathrm{s}}$, diverse shapes of the SMS can be obtained. In 
order to get a better insight into the change behavior of pore size during the shape change, Micro-XCT was applied and the results are displayed in Fig. 3. The black background is clearly seen across the SMS when it is in the initial state, demonstrating that the SMS has the open-pore structure (Fig. 3a). In the pressed state, the frameworks of the SMS are overlapped; however, small black background can still be seen across the SMS, demonstrating that even in the pressed state with $R_{\mathrm{s}}=0 \%$, the SMS still has some interconnected pore structures (Fig. 3b). As the SMS is recovered from the pressed state to the original state, the pore structure becomes more and more clearly (Fig. 3c-f), and when the $R_{\mathrm{s}}=100 \%$, the pore structure seems the same with the original state. The pore size was also examined, which can be precisely adjusted from about $28 \mathrm{~nm}$ to $895 \mu \mathrm{m}$ with the increase of $R_{\mathrm{s}}$ (Fig. 3g). It should be noted that the change of pore size has no influence on the wettability of PPy surface. When the SMS resides at a random state with a random pore size, switchable superhydrophobicity/superhydrophilicity can be always achieved as the PPy is repeatedly oxidized and reduced (Fig. 3h, the bottom surface always keeps high hydrophilicity during the variation of shape, Fig. S15). By using the sponge as a permeation film, as shown in Fig. S16, ON/OFF water permeation can be reversibly controlled due to the switchable wetting performance. More importantly, such $\mathrm{ON} / \mathrm{OFF}$ control can be always achieved when the sponge resides in a random state and displays a random pore size (Fig. 3i). Besides the ON/OFF permeation control, it is found that when the PPy is in the superhydrophilic state, the permeation flux can be exactly controlled by tuning the pore size, which is increased with increasing pore size. These results demonstrate that based on the cooperative
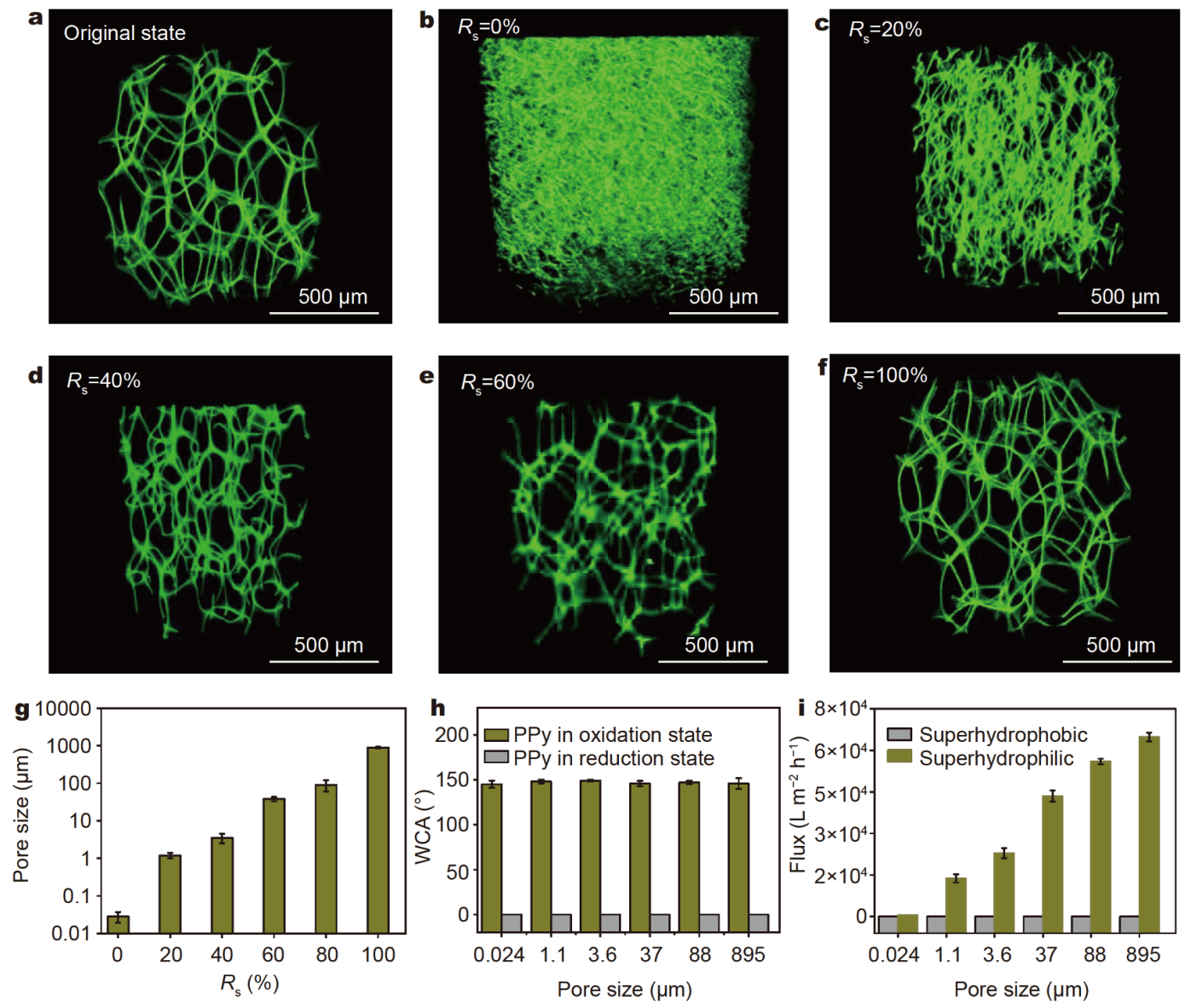

Figure 3 Typical 3D-reconstructed Micro-XCT images of SMS at different states: (a) initial, (b) after pressing, (c-f) corresponding to the different $R_{\mathrm{s}}$. (g) Statistic of the pore size of the SMS as the $R_{\mathrm{s}}$ is increased, indicating that the pore size can be accurately regulated. (h) Statistic of the top surface WCA on the sponge with different pore sizes, meaning that the switchable superhydrophobicity/superhydrophilicity can be always observed regardless of the variation of pore size. (i) Statistic of the water permeation flux when the sponge shows different wettabilities and pore sizes, demonstrating that not only ON/OFF water permeation, but also precise permeation flux can be controlled. 
effect between smart surface wettability and tunable pore size, ON/OFF water permeation with simultaneous accurate permeation flux can be acquired (Fig. 3i). This feature is apparently superior to previous smart superwetting porous materials that only have a constant permeation flux due to the static porous structure [3$5,17,18]$.

To sum up, it can be noted that good controllability of pore size and surface wettability is crucial for the excellent water permeation control, and the inner mechanism can be explained as follows. It is well known that TPI is a type of SMP (Fig. S17), which can provide the SMS with the SME $[55,56]$. Briefly, the original sponge with a larger pore size (about $895 \mu \mathrm{m}$ ) and thickness is the permanent shape (upper left in Fig. 1b). In this status, the conformation of the TPI molecular chain has the highest entropy and resides in the thermodynamically stable state (bottom left in Fig. 1b). Meanwhile, the relatively high modulus of TPI can fix the PU sponge's shape and pore size (Fig. S18). As the SMS is heated to a temperature higher than the melt temperature $\left(T_{\mathrm{m}}\right)$ of the TPI, the crystalline areas of the TPI would be melted, and TPI becomes soft (Fig. S17). In this case, the shape of the SMS can be tuned to a new shape under the pressure. In this process, molecular chain conformation of the TPI would transform to a lower-entropy state. As the crystalline areas in TPI is reformed under cooling (bottom right in Fig. 1b), the relatively high modulus of the TPI can be regained. The high modulus of TPI can overcome the resilience of the PU sponge (upper right in Fig. 1b), and thus the temporary pressed shape of the SMS can be kept. Due to the compression, the framework of the sponge would be overlapped, the average pore size of the SMS is decreased, forming the nanopores eventually (about $28 \mathrm{~nm}$ ). When the SMS is reheated (higher than $T_{\mathrm{m}}$ ), the crystalline regions would be melted again, and TPI molecular chains will be reactivated. In this case, the tendency to increase entropy make the molecular chain conformation to return to the permanent state, and with the help of the resilience of the PU sponge substrate, the sponge shape and pore size can spontaneously recover to the original state. After further cooling to room temperature, the crystalline domains would be reformed, and finally, the microscale pore size of the SMS can be fixed again. Because the crystalline region in TPI can be formed/melted repeatedly, the pore size and shape of the SMS can be changed alternately.

The switchable wetting performance with a high contrast on the PPy surface was attributed to the rough sponge surface structure as well as the doping/de-doping of PFOS ions under different redox states. Because the PFOS ions have a low surface energy, the PPy layer doped with PFOS ions exhibits hydrophobicity, after de-doping of the PFOS ions, it would display the hydrophilicity $[10,15]$. The reduction potential can drive the PFOS ions out of the PPy layer, while an oxidation potential can lead to a reverse process (Fig. S19); together with the enhanced effect of the rough structure on sponge itself $[57,58]$, superhydrophobic/superhydrophilic transition can be achieved. When the PPy surface shows the superhydrophobicity, the excellent water-repellent ability would restrain the water from wetting/permeating the SMS, and thus as shown in Fig. 3i, regardless of pore size, water cannot pass the sponge and the flux is zero. When the PPy surface converts to be superhydrophilic, because the SMS is also hydrophilic due to the presence of PDA/ PEI (Fig. S15), water can pass through the whole sponge spontaneously under its gravity. In this condition, the flux can be adjusted by regulating the pore size, which is described by the Hagen-Poiseuille equation [59]:

$J=\frac{p \pi r 2 \Delta P}{8 \mu L \tau}$,

where the flux was marked as $J$, the porosity was marked as $p$, the pore radius was marked as $r$, the pressure drop was marked as $\Delta P$, the liquid viscosity was marked as $\mu$, the thickness of SMS was marked as $L$, and the tortuosity of the SMS was marked as $\tau$. From Equation (2), we can find that permeation flux will be increased following the increase of $p$ and $r$, and it will be decreased as $L$ increases. Herein, the change of $r$ is three orders of magnitude more than that of $L$, and the change of $p$ and $r$ determines the permeation flux of the liquid. Because $p$ and $r$ can be controlled precisely by regulating $R_{\mathrm{s}}$ (Fig. $3 \mathrm{~g}$ and Fig. S20), as displayed in Fig. 3i, the permeation flux can also be accurately regulated. From the above, it would be easy to understand that the switchable wettability and tunable pore size provide the sponge with smart ON/OFF water permeation and accurate flux, respectively. Herein, it is worth noting that the initial thickness of the SMS is also important, to obtain a wide range regulation of pore size from nanoscale to microscale; herein, it is found that the minimal thickness is about $3 \mathrm{~cm}$ (Fig. S21).

The obtained SMS has so distinguished controllability in water permeation based on excellent tunability in both pore size and surface wettability, indicating that it can be applied in various research areas, for instance as a system for adjusting the small molecule release. Nowadays, porous materials with adjustable small molecule release property have aroused great interest [60-62]. However, most of current porous materials cannot regulate the 
release velocity and only realize simple ON/OFF release [63-65]. Herein, based on our sponge, diverse release manners can be achieved. To demonstrate such advantages, a small molecule release system was prepared by mounting the sponge at the bottom of a container. Rhodamine B (Rh B) aqueous solution and pure water were put in and outside of the vessel, respectively, and the concentration gradient of $\mathrm{Rh} \mathrm{B}$ compels them to pass through the SMS to reach the water container (Fig. S22). When the PPy is in the reduction state with superhydrophilicity, it can be seen that as the releasing time is increased, the concentration of $\mathrm{Rh} \mathrm{B}$ outside the vessel is increased, and finally reaches a plateau, which is about $90 \%$ relative to the original concentration. Moreover, as the pore diameter is increased by increasing the $R_{\mathrm{s}}$ (Fig. $4 \mathrm{a}-\mathrm{c}$ ), the needed time to reach the plateau is decreased, indicating that the release rate is accelerated significantly. These results prove that accurate release velocity can be achieved based on the tunability in pore diameter. When the PPy is in the oxidation state with superhydrophobicity (Fig. $4 \mathrm{~d}-\mathrm{f}$ ), it can be seen that the release velocity is extremely slow, since the air trapped among the superhydrophobic microstructures needs a long time to be replaced by water [34], and thus the sponge can be used for long-term sustained release. Certainly, by adjusting the pore size, the release velocity can also be further regulated, which is also increased as the $R_{\mathrm{s}}$ increases. Herein, it should be noted that the maximal release concentration is about $70 \%$ of the original value, which is apparently lower compared with that when the PPy is in the reduction state. This difference can be ascribed to that more $\mathrm{Rh} \mathrm{B}$ molecules can be adsorbed by the PPy film when it is in the oxidation state since the PFOS ions have the opposite charge with the Rh B [10]. Fig. 4g-i indicate the development of the accumulative concentration of Rh B with increasing time as the PPy is
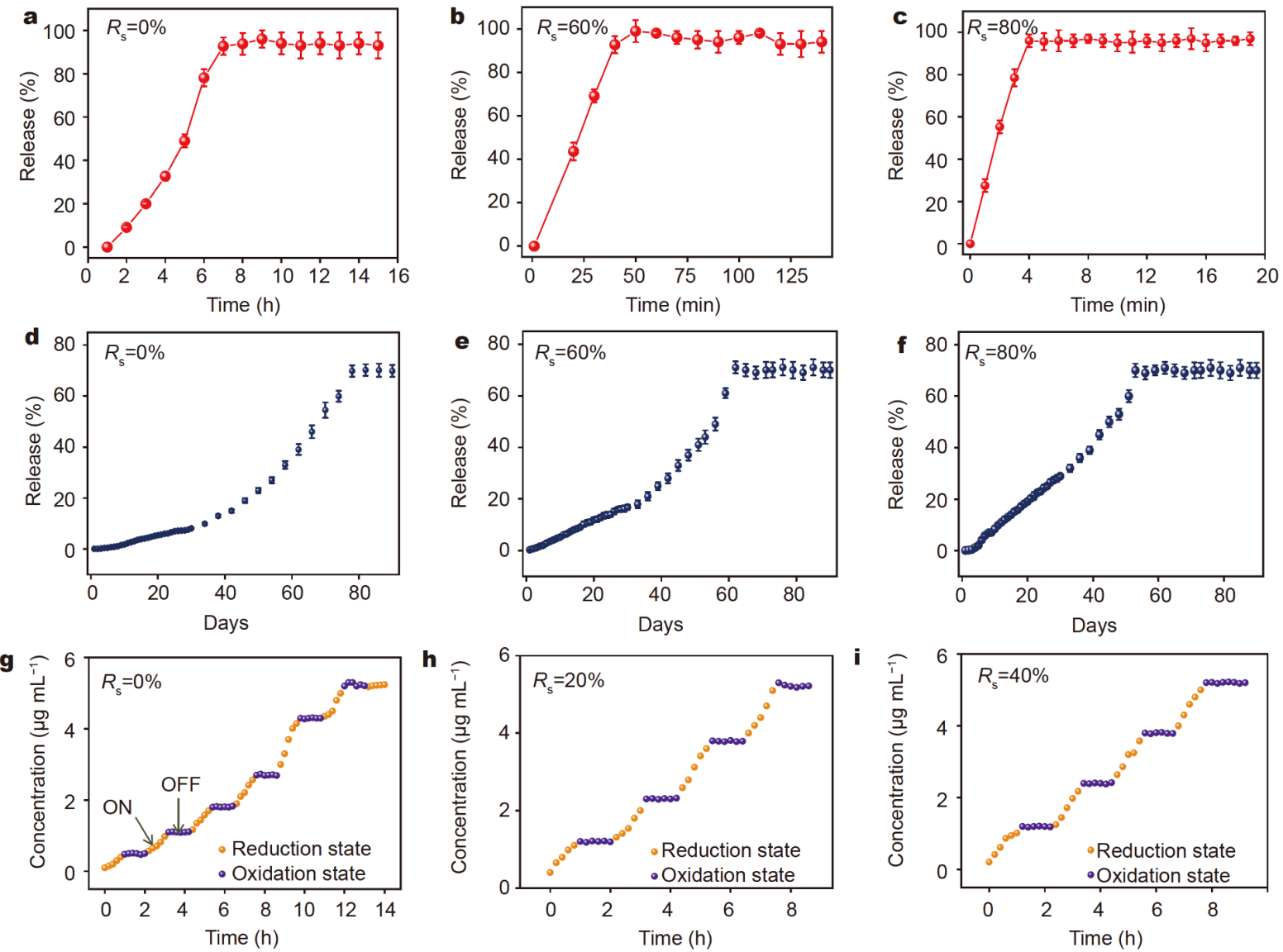

Figure 4 Application of the sponge in controlling the molecule release: cumulative release of Rh B when the sponge shows the superhydrophilicity with different pore sizes $(a-c)$, the superhydrophobicity with different pore sizes $(d-f)$, and the switching superhydrophobicity/superhydrophilicity with different pore sizes $(\mathrm{g}-\mathrm{h})$. These results demonstrate that through regulating surface wettability and pore size, diverse release manners with controlled release velocity can be achieved. 
reduced and oxidized alternately. It can be seen that the concentration of Rh B is increased when the PPy is in the reduction state. However, when PPy is in the oxidation state, the concentration remains constant overtime, which indicates that no $\mathrm{Rh} \mathrm{B}$ penetrated through the sponge in a short time, demonstrating a pulsatile release of the small molecules. Similar with the above results, by adjusting $R_{s}$, the release velocity in every stage can also be controlled. From the above, it can be found that diverse release manners including fast release, pulsatile release, and sustained release can be achieved; as for every release manner, by simply regulating the pore size, the release velocity can be further precisely controlled, demonstrating an excellent controllability in molecule release. Moreover, the small molecule release is only a model; by means of the excellent controllability in surface wettability and pore size, the modified sponge can also be potentially used in many other applications, such as precise chemical synthesis, accurate drug release, and microfluidic devices.

\section{CONCLUSIONS}

In summary, the porous sponge with tunability in both surface wettability and pore size is prepared by electrodepositing a layer of responsive PPy coating on the SMS. The doping/de-doping the PFOS ions on the PPy coating provides the sponge surface with switchable superhydrophobicity/superhydrophilicity, and the SMS offers the adjustable pore size from about $28 \mathrm{~nm}$ to $895 \mu \mathrm{m}$. Through synergistically regulating the surface wettability and pore size, ON/OFF water permeation with simultaneously accurate permeation flux can be achieved. Moreover, the sponge was further applied in molecule release, and diverse release manners including fast release, sustained release and pulse release with accurate release velocity can be realized. This paper reports a new design method based on the combination of shape memory pore structure with responsive molecules, which successfully eliminates the imperfection present in existing porous materials in which only single surface wettability or pore size can be controlled, and meanwhile improves the functions of the smart porous materials. Moreover, the design method is so simple that it is believed that except $\mathrm{PPy}$, a lot of other stimuli-responsive molecules may also be applied to synthesize more smart porous materials, such as those responsive to $\mathrm{pH}$ and light.

Received 23 November 2020; accepted 7 January 2021; published online 26 March 2021

1 Yao X, Hu Y, Grinthal A, et al. Adaptive fluid-infused porous films with tunable transparency and wettability. Nat Mater, 2013, 12: 529-534

2 Wang S, Liu K, Yao X, et al. Bioinspired surfaces with superwettability: New insight on theory, design, and applications. Chem Rev, 2015, 115: 8230-8293

3 Gao X, Xu LP, Xue Z, et al. Dual-scaled porous nitrocellulose membranes with underwater superoleophobicity for highly efficient oil/water separation. Adv Mater, 2014, 26: 1771-1775

4 Lv W, Mei Q, Xiao J, et al. 3D multiscale superhydrophilic sponges with delicately designed pore size for ultrafast oil/water separation. Adv Funct Mater, 2017, 27: 1704293

5 Chu Z, Feng Y, Seeger S. Oil/water separation with selective superantiwetting/superwetting surface materials. Angew Chem Int Ed, 2015, 54: 2328-2338

6 Yu C, Zhang P, Wang J, et al. Superwettability of gas bubbles and its application: From bioinspiration to advanced materials. Adv Mater, 2017, 29: 1703053

7 Chen C, Huang Z, Shi L, et al. Remote photothermal actuation of underwater bubble toward arbitrary direction on planar slippery $\mathrm{Fe}_{3} \mathrm{O}_{4}$-doped surfaces. Adv Funct Mater, 2019, 29: 1904766

8 Ning Y, Zhang D, Ben S, et al. An innovative design by single-layer superaerophobic mesh: Continuous underwater bubble antibuoyancy collection and transportation. Adv Funct Mater, 2020, 30: 1907027

9 Song Y, Roy P, Paramasivam I, et al. Voltage-induced payload release and wettability control on $\mathrm{TiO}_{2}$ and $\mathrm{TiO}_{2}$ nanotubes. Angew Chem, 2010, 122: 361-364

10 Zhang Q, Kang J, Xie Z, et al. Highly efficient gating of electrically actuated nanochannels for pulsatile drug delivery stemming from a reversible wettability switch. Adv Mater, 2018, 30: 1703323

11 Cai Q, Qiao C, Ning J, et al. A polysaccharide-based hydrogel and PLGA microspheres for sustained P24 peptide delivery: An in vitro and in vivo study based on osteogenic capability. Chem Res Chin Univ, 2019, 35: 908-915

$12 \mathrm{Hu} \mathrm{Q}$, Li G, Liu X, et al. Superhydrophilic phytic-acid-doped conductive hydrogels as metal-free and binder-free electrocatalysts for efficient water oxidation. Angew Chem, 2019, 131: 4362-4366

$13 \mathrm{Li} \mathrm{J}$, Gao X, Li Z, et al. Superhydrophilic graphdiyne accelerates interfacial mass/electron transportation to boost electrocatalytic and photoelectrocatalytic water oxidation activity. Adv Funct Mater, 2019, 29: 1808079

14 Yu X, Wang Z, Jiang Y, et al. Reversible pH-responsive surface: From superhydrophobicity to superhydrophilicity. Adv Mater, 2005, 17: 1289-1293

$15 \mathrm{Xu} \mathrm{L}$, Chen W, Mulchandani A, et al. Reversible conversion of conducting polymer films from superhydrophobic to superhydrophilic. Angew Chem Int Ed, 2005, 44: 6009-6012

16 Lim HS, Kwak D, Lee DY, et al. UV-driven reversible switching of a roselike vanadium oxide film between superhydrophobicity and superhydrophilicity. J Am Chem Soc, 2007, 129: 4128-4129

17 Pan $\mathrm{Z}$, Cao S, Li J, et al. Anti-fouling $\mathrm{TiO}_{2}$ nanowires membrane for oil/water separation: Synergetic effects of wettability and pore size. J Membrane Sci, 2019, 572: 596-606

18 Ou R, Wei J, Jiang L, et al. Robust thermoresponsive polymer composite membrane with switchable superhydrophilicity and superhydrophobicity for efficient oil-water separation. Environ Sci Technol, 2016, 50: 906-914

19 Zhang W, Liu N, Zhang Q, et al. Thermo-driven controllable emulsion separation by a polymer-decorated membrane with switchable wettability. Angew Chem Int Ed, 2018, 57: 5740-5745 
20 Chen L, Wang W, Su B, et al. A light-responsive release platform by controlling the wetting behavior of hydrophobic surface. ACS Nano, 2014, 8: 744-751

21 Yan $\mathrm{T}$, Chen $\mathrm{X}$, Zhang $\mathrm{T}$, et al. A magnetic $\mathrm{pH}$-induced textile fabric with switchable wettability for intelligent oil/water separation. Chem Eng J, 2018, 347: 52-63

22 Xu Y, Zhang Z, Geng X, et al. Smart carbon foams with switchable wettability for fast oil recovery. Carbon, 2019, 149: 242-247

23 Cheng M, Liu Q, Ju G, et al. Bell-shaped superhydrophilic-superhydrophobic-superhydrophilic double transformation on a $\mathrm{pH}$ responsive smart surface. Adv Mater, 2014, 26: 306-310

24 Wang J, Sun L, Zou M, et al. Bioinspired shape-memory graphene film with tunable wettability. Sci Adv, 2017, 3: e1700004

25 Gao W, Wang J, Zhang X, et al. Electric-tunable wettability on a paraffin-infused slippery pattern surface. Chem Eng J, 2020, 381: 122612

26 MacDiarmid AG. "Synthetic metals": A novel role for organic polymers. Angew Chem Int Ed, 2001, 40: 2581-2590

27 Lim HS, Lee SG, Lee DH, et al. Superhydrophobic to superhydrophilic wetting transition with programmable ion-pairing interaction. Adv Mater, 2008, 20: 4438-4441

28 Sheng $\mathrm{Z}$, Wang $\mathrm{H}$, Tang $\mathrm{Y}$, et al. Liquid gating elastomeric porous system with dynamically controllable gas/liquid transport. Sci Adv, 2018, 4: eaao6724

29 Zhao Y, Wang H, Zhou H, et al. Directional fluid transport in thin porous materials and its functional applications. Small, 2017, 13: 1601070

30 Cai Y, Lin L, Xue Z, et al. Filefish-inspired surface design for anisotropic underwater oleophobicity. Adv Funct Mater, 2014, 24: 809-816

31 Liu Y, Su Y, Guan J, et al. 2D heterostructure membranes with sunlight-driven self-cleaning ability for highly efficient oil-water separation. Adv Funct Mater, 2018, 28: 1706545

32 Li F, Wang Z, Huang S, et al. Flexible, durable, and unconditioned superoleophobic/superhydrophilic surfaces for controllable transport and oil-water separation. Adv Funct Mater, 2018, 28: 1706867

$33 \mathrm{Gu}$ J, Fan H, Li C, et al. Robust superhydrophobic/superoleophilic wrinkled microspherical MOF@rGO composites for efficient oilwater separation. Angew Chem, 2019, 131: 5351-5355

34 Yohe ST, Colson YL, Grinstaff MW. Superhydrophobic materials for tunable drug release: Using displacement of air to control delivery rates. J Am Chem Soc, 2012, 134: 2016-2019

35 Wang $\mathrm{D}$, Guo Z, Chen $\mathrm{Y}$, et al. In situ hydrothermal synthesis of nanolamellate $\mathrm{CaTiO}_{3}$ with controllable structures and wettability. Inorg Chem, 2007, 46: 7707-7709

$36 \mathrm{Xu} \mathrm{YF,} \mathrm{Ma} \mathrm{DK,} \mathrm{Chen} \mathrm{XA,} \mathrm{et} \mathrm{al.} \mathrm{Bisurfactant-controlled} \mathrm{synthesis}$ of three-dimensional $\mathrm{YBO}_{3} / \mathrm{Eu}^{3+}$ architectures with tunable wettability. Langmuir, 2009, 25: 7103-7108

37 Liu P, Lai H, Luo X, et al. Superlyophilic shape memory porous sponge for smart liquid permeation. ACS Nano, 2020, 14: 1404714056

38 Zhang D, Cheng Z, Kang H, et al. A smart superwetting surface with responsivity in both surface chemistry and microstructure. Angew Chem, 2018, 130: 3763-3767

39 Cheng Z, Zhang D, Lv T, et al. Superhydrophobic shape memory polymer arrays with switchable isotropic/anisotropic wetting. Adv Funct Mater, 2018, 28: 1705002

40 Cheng Z, Zhang D, Luo X, et al. Superwetting shape memory microstructure: Smart wetting control and practical application. Adv Mater, 2021, 33: 2001718
41 Lin C, Lv J, Li Y, et al. 4D-printed biodegradable and remotely controllable shape memory occlusion devices. Adv Funct Mater, 2019, 29: 1906569

42 Lendlein A, Langer R. Biodegradable, elastic shape-memory polymers for potential biomedical applications. Science, 2002, 296: 1673-1676

43 Chan BQY, Low ZWK, Heng SJW, et al. Recent advances in shape memory soft materials for biomedical applications. ACS Appl Mater Interfaces, 2016, 8: 10070-10087

44 Fang Y, Ni Y, Choi B, et al. Chromogenic photonic crystals enabled by novel vapor-responsive shape-memory polymers. Adv Mater, 2015, 27: 3696-3704

45 Wang W, Shen D, Li X, et al. Light-driven shape-memory porous films with precisely controlled dimensions. Angew Chem, 2018, 130: $2161-2165$

46 Jiang $\mathrm{S}, \mathrm{Hu} \mathrm{Y}, \mathrm{Wu} \mathrm{H}$, et al. Multifunctional Janus microplates arrays actuated by magnetic fields for water/light switches and bioinspired assimilatory coloration. Adv Mater, 2019, 31: 1807507

47 Chen CM, Yang S. Directed water shedding on high-aspect-ratio shape memory polymer micropillar arrays. Adv Mater, 2014, 26: 1283-1288

48 Yao X, Dunn SS, Kim P, et al. Fluorogel elastomers with tunable transparency, elasticity, shape-memory, and antifouling properties. Angew Chem Int Ed, 2014, 53: 4418-4422

49 Song J, Gao M, Zhao C, et al. Large-area fabrication of droplet pancake bouncing surface and control of bouncing state. ACS Nano, 2017, 11: 9259-9267

50 Bai X, Yang Q, Fang Y, et al. Anisotropic, adhesion-switchable, and thermal-responsive superhydrophobicity on the femtosecond laserstructured shape-memory polymer for droplet manipulation. Chem Eng J, 2020, 400: 125930

51 Lv T, Cheng Z, Zhang D, et al. Superhydrophobic surface with shape memory micro/nanostructure and its application in rewritable chip for droplet storage. ACS Nano, 2016, 10: 9379-9386

52 Gui X, Wei J, Wang K, et al. Carbon nanotube sponges. Adv Mater, 2010, 22: 617-621

53 Cui Y, Wang Y, Shao Z, et al. Smart sponge for fast liquid absorption and thermal responsive self-squeezing. Adv Mater, 2020, 32: 1908249

54 Barrett EP, Joyner LG, Halenda PP. The determination of pore volume and area distributions in porous substances. I. Computations from nitrogen isotherms. J Am Chem Soc, 1951, 73: 373-380

55 Xia L, Wu H, Qiu G. Shape memory behavior of carbon nanotubereinforced trans-1,4-polyisoprene and low-density polyethylene composites. Polym Adv Technol, 2020, 31: 107-113

56 Tsujimoto $\mathrm{T}$, Toshimitsu K, Uyama $\mathrm{H}$, et al. Maleated trans-1,4polyisoprene from Eucommia ulmoides Oliver with dynamic network structure and its shape memory property. Polymer, 2014, 55: 6488-6493

57 Su B, Tian Y, Jiang L. Bioinspired interfaces with superwettability: From materials to chemistry. J Am Chem Soc, 2016, 138: 17271748

58 Min WL, Jiang B, Jiang P. Bioinspired self-cleaning antireflection coatings. Adv Mater, 2008, 20: 3914-3918

59 Karan S, Samitsu S, Peng X, et al. Ultrafast viscous permeation of organic solvents through diamond-like carbon nanosheets. Science, 2012, 335: 444-447

60 Dong Z, Sun Y, Chu J, et al. Multivariate metal-organic frameworks for dialing-in the binding and programming the release of drug molecules. J Am Chem Soc, 2017, 139: 14209-14216 
61 Tokarev I, Minko S. Stimuli-responsive porous hydrogels at interfaces for molecular filtration, separation, controlled release, and gating in capsules and membranes. Adv Mater, 2010, 22: 34463462

62 Carrillo-Carrión C, Martínez R, Navarro Poupard MF, et al. Aqueous stable gold nanostar/ZIF-8 nanocomposites for lighttriggered release of active cargo inside living cells. Angew Chem, 2019, 131: 7152-7156

63 Nagata S, Kokado K, Sada K. Metal-organic framework tethering PNIPAM for ON-OFF controlled release in solution. Chem Commun, 2015, 51: 8614-8617

64 Mal NK, Fujiwara M, Tanaka Y. Photocontrolled reversible release of guest molecules from coumarin-modified mesoporous silica. Nature, 2003, 421: 350-353

65 Ogoshi T, Takashima S, Yamagishi TA. Photocontrolled reversible guest uptake, storage, and release by azobenzene-modified microporous multilayer films of pillar[5]arenes. J Am Chem Soc, 2018, 140: $1544-1548$

Acknowledgements This work was supported by the National Natural Science Foundation of China (22075061, 21674030 and 51790502).

Author contributions Liu P and Lai $\mathrm{H}$ carried out the investigation and wrote the paper; Xia Q and Zhang D carried out the mechanical measurements and theoretical analysis; Cheng Z, Liu Y and Jiang L contributed to the conceptualization, supervision and valuable discussion.

Conflict of interest The authors declare that they have no conflict of interest.

Supplementary information Supporting data are available in the online version of the paper.

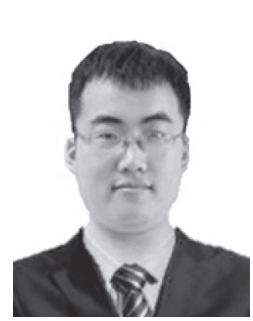

Pengchang Liu received his Bachelor's degree from Harbin University of Science and Technology in 2012, and his Master's degree at Jilin University in 2015. He is currently a PhD student at the School of Chemistry and Chemical Engineering, Harbin Institute of Technology, China. His present research interest focuses on porous shape memory polymers with liquid permeation.

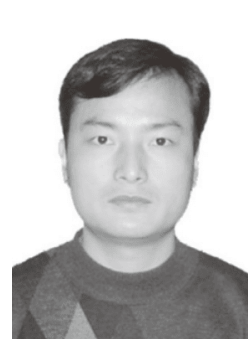

Zhongjun Cheng obtained his BSc (2003) and MSc (2006) degrees in chemistry at Jilin University, China, and his $\mathrm{PhD}$ degree (2009) at the Institute of Chemistry, Chinese Academy of Sciences, under the supervision of Professor Lei Jiang. He is currently an associate professor at Harbin Institute of Technology, Heilongjiang, China. His scientific interest is in the design and fabrication of superwetting materials with dynamic tunable micro-/nanostructures, and related applications.

\section{表面浸润性和孔径可调控的多孔形状记忆海绵用 于智能分子释放}

刘鹏昌 $^{1 \dagger}$, 来华 ${ }^{1 \dagger}$, 夏琦兴 ${ }^{3}, 弓$ 东杰 $^{1}$, 成中军 ${ }^{1^{*}}$, 刘宇艳 ${ }^{1^{*}}$, 江雷 ${ }^{2}$

摘要 近年来, 智能超浸润多孔材料受到了广泛关注. 该材料的孔 径和表面浸润性是决定其功能的关键因素. 然而, 目前已报道的材 料多数仅采用单一的调控手段, 从而大大限制了材料的可控性、 功能和应用. 我们将聚吡咯通过电沉积的方式修饰在形状记忆海 绵的表面上, 可以实现表面浸润性从超亲水到超疏水、孔径从 $895 \mu \mathrm{m}$ 到 $28 \mathrm{~nm}$ 的切换. 通过协同调控表面浸润性和孔径大小, 可 以实现对水渗透的开关控制以及流速的精确控制. 同时, 该材料还 可以应用于智能分子释放实现精确可控的多重释放方式. 本文报 道的表面浸润性和孔径均可调的超浸润多孔材料, 为超浸润多孔 材料的设计提供了一种新思路. 